\title{
XLVIII. On the theory of illumination in a fog
}

\section{Lord Rayleigh F.R.S.}

To cite this article: Lord Rayleigh F.R.S. (1885) XLVIII. On the theory of illumination in a fog , Philosophical Magazine Series 5, 19:121, 443-446, DOI: 10.1080/14786448508627698

To link to this article: http://dx.doi.org/10.1080/14786448508627698

册 Published online: 29 Apr 2009.

Submit your article to this journal 준

Џll Article views: 6

Q View related articles $₫$ 
I need hardly say, in conclusion, that I do not in the least. intend to convey the impression that the actual structure of the æther is a bit like what I have described. What physicists ought to look for is such a mode of motion in space as will confer upon it the properties required in order that it may exhibit electromagnetic phenomena. Such a mode of motion would be a real explanation of these phenomena. I have only given a description of them.

I think, however, that it is worth while considering these models, because in them the disturbance which represents light is not the same as the vibrations of an elastic jelly, for what represents an electric displacement is a change of structure of an element, and not a displacement of the element; and it seems almost certain that, notwithstanding the very high authority which seems to support the view that the æther is like an elastic jelly, nevertheless its vibrations are much more of the nature of alterations in structure than of displacements.

XLVIII. On the Theory of Illumination in a Fog. By Lord Rayleigh, F.R.S.*

A $S$ a step towards a better understanding of the action of A fog upon light, it seems desirable to investigate what the phenomena would be in the simplest case that can be proposed. For this purpose we may consider the atmosphere and the material composing the fog to be absolutely transparent, and also make abstraction from the influence of obstacles, among which must be included the ground itself.

Conceive a small source of radiation, $e . g$. an incandescent carbon filament, to be surrounded by a spherical clond, of uniform density, or at any rate symmetrically disposed round the source, outside of which the atmosphere is clear. Since by hypothesis there is no absorption, whatever radiation is emitted by the source passes outward through the external surface of the cloud. The effect of the cloud is to cause diffusion, $i . e$. to spread the rays passing through any small area of the surface (which in the absence of the cloud would be limited to a small solid angle) more or less uniformly over the complete hemisphere.

Whether the total radiation passing outwards through the small area on the external surface of the cloud is affected by the existence of the cloud depends upon the circumstances of the case. If it be laid down that the total emission of energy from the source is given, then the presence of the cloud

* Communicated by the Physical Society : read April 25, 1885. 
makes no difference in respect of the energy passing any element of the spherical area. But this supposition does not correspond to a constant temperature of the source, in consequence of the energy received back from the cloud by reflection. To keep the total emission of energy constant, we should have to suppose a rise of temperature increasing indefinitely with the size and density of the cloud.

Let us now suppose that the region under consideration is bounded upon all sides by a distant envelope of perfect reflecting-power. Then, whatever the density of the clouds which may wholly or partially occupy the enclosure, we know, by the second law of thermodynamics, that at every internal point there is radiation in every direction of the full amount corresponding to the temperature of the source. In one sense this conclusion holds good, even although the matter composing the cloud has the power of absorption. But in that case equilibrium would not be attained until the clouds themselves to the remotest parts had acquired the temperature of the source; whereas under the supposition of perfect transparency the temperature of the cloud is a matter of indifference; and equilibrium is attained in a time dependent upon that required by light to traverse the enclosure. So far we have made no supposition as to the distribution of the cloud; but we will now imagine a layer of such thickness as to allow only a very small fraction of the incident radiation to penetrate it, to line the interior of the reflecting envelope. This layer itself plays the part of a practically perfect reflector; and it is not difficult to see that the reflecting envelope hitherto conceived to lie beyond it may be removed without interfering with the state of things on the inner side of the layer of cloud. We thus arrive at the rather startling conclusion that at any distance from the source, and whatever the distribution of clouds, there is always in every direction the full radiation due to the temperature of the source, provided only that there lie outside a complete sbell of cloud sufficiently thick to be impervious. And this state of things is maintained without (on the whole) emission of energy from the source.

Even if the material composing the cloud possesses absorbing-power for some kinds of radiation, e.g. for dark radiation, but is perfectly transparent to other kinds, e.g. luminous radiation, the general theorem holds good as respects the latter kinds; so that in the case supposed the light would still be everywhere the same as in a clear enclosure whose walls have throughout the same luminosity as the source. But in order to compensate the absorption of dark rays, the source must now be supplied with energy. 
Some of the principles here enunciated have an acoustical as well as an optical application, and indeed first occurred to me some years ago in connection with Prof. Tyndall's investigations upon fog-signals. The effect of "acoustic clouds" analogous to fog (and unattended with absorption of energy), might be very different upon the report of a gun and upon the sustained sound of a siren, the latter being reinforced by reflection from the acoustic fog.

The theory presented in the present paper may be illustrated by the known solution of the comparatively simple problem of a pile of transparent plates*. If $\rho$ denote the proportion of the incident light reflected at a single surface, then the proportion reflected $\phi(m)$, and transmitted $\psi(m)$, by a pile of $m$ plates is given by

$$
\frac{\phi(m)}{2 m \rho}=\frac{\psi(m)}{1-\rho}=\frac{1}{1+(2 m-1) \rho} .
$$

From these expressions it is evident that, however small $\rho$ may be, $i$. e. however feeble the reflection at a single surface, we have only to suppose $m$ large enough in order that the reflection may be as complete, and the transmission as small, as we please. Such a pile may, under ordinary conditions, be regarded as impervious.

But now suppose that after passing the pile of $m$ plates, the light is incident upon a second pile of $n$ plates, and consider the intensity between the two piles, the original intensity being unity, as before. For the intensity of the light travelling in the original direction we have

$$
\begin{gathered}
\psi(m)+\psi(m) \cdot \phi(n) \cdot \phi(m)+\psi(m) \cdot\{\phi(n) \cdot \phi(m)\}^{2} \\
+\psi(m) \cdot\{\phi(n) \cdot \phi(m)\}^{3}+\ldots ;
\end{gathered}
$$

or on summation of the geometric series,

$$
\frac{\psi(m)}{1-\phi(n) \cdot \phi(m)}
$$

If we introduce the values of $\phi$ and $\psi$ in terms of $m, n, \rho$, this becomes

$$
\frac{2 n \rho+1-\rho}{2(m+n) \rho+1-\rho} \text {. }
$$

In like manner, for the light going the other way we have

$$
\frac{\phi(m) \cdot \phi(n)}{1-\phi(m) \cdot \phi(n)} ;
$$

* Stokes, Proc. Roy. Soc. vol. xi. p. 545 (1862).

Phil. Mag. S. 5. Vol. 19. No. 121. June 1885. $2 \mathrm{H}$ 
or in terms of $m, n, \rho$,

$$
\frac{2 n \rho}{2(m+n) \rho+1-\rho} .
$$

When $m$ and $n$ are great, both expressions reduce to $n /(m+n)$; so that the light passing in the two directions is equally bright. Moreover, and this is the point to be especially noticed, however great $m$ may be-that is, however impervious the first pile is, the light between the two piles may be made to approach the original light in brightness as nearly as we please, by sufficiently increasing the number of plates in the second pile; that is, the light between the piles may be made to be the same as if the first pile were removed. From this example we may understand more clearly how a very small quantity of light penetrating directly may be beaten backwards and forwards, as between two reflectors, until the original intensity is recovered.

XLIX. A Monochromatic Telescope, with Application to Photometry. By Lord RAYLEIGH, F.R.S.*

THE purpose of this instrument is to exhibit external objects 1 as they would be seen either with the naked eye, or through a telescope, if lighted with approximately monochromatic light; that is, to do more perfectly what is done roughly by a coloured glass.

The arrangement is not new, though I am not aware that it has ever been described. In 1870 I employed it for determinations of absorption, and, if my memory serves me right, I heard soon afterwards from Clerk-Maxwell that he also had used it. It is, indeed, a very slight modification of Maxwell's colour-box.

In the ordinary form of that instrument, white light admitted through a slit is rendered parallel by a collimating lens, dispersed by flint-glass prisms, and then brought to a focus at a screen, upon which accordingly a pure spectrum is formed. This screen is perforated by a second slit, immediately behind which the observer places his eye. It is evident that the light passing the aperture is approximately monochromatic, so that the observer, if he focuses his eye suitably, will see the prism illuminated with this kind of light. The only addition now required to convert the instrument into a monochromatic telescope is a lens placed just within the first slit, of such power as to throw an image of external objects

* Communicated by the Physical Society : read April 25, 1885. 